\title{
AKT/mTOR signaling pathway is involved in salvianolic acid B-induced autophagy and apoptosis in hepatocellular carcinoma cells
}

\author{
LING GONG ${ }^{1}$, CHUNHONG DI $^{2}$, XIAOFANG XIA ${ }^{1}$, JIE WANG $^{1}$, GONGYING CHEN $^{1}$, \\ JUNPING SHI $^{1}$, PENGSHUAI CHEN ${ }^{3}$, HUI XU ${ }^{3}$ and WEIBING ZHANG ${ }^{3}$ \\ Departments of ${ }^{1}$ Liver Diseases and ${ }^{2}$ Laboratory, The Affiliated Hospital of Hangzhou Normal University, \\ Hangzhou, Zhejiang 310015; ${ }^{3}$ Department of Pharmacy, The First Affiliated Hospital \\ of Wenzhou Medical University, Wenzhou, Zhejiang 325000, P.R. China
}

Received July 6, 2016; Accepted October 17, 2016

DOI: $10.3892 /$ ijo.2016.3748

\begin{abstract}
Chinese medicines are emerging as an attractive new generation of anticancer drugs. Here, we explored the impact of salvianolic acid B (Sal B), the major water-soluble compounds of Danshen, on apoptosis and autophagy of human hepatocellular carcinoma cells (HCC). We also investigated the related molecular mechanisms. We found that Sal B exhibits potent ability to inhibit HCC cells viability in a concentration-dependent manner, and to induce apoptosis via the mitochondrial apoptosis pathway. Additionally, Sal B could also induce autophagy. Furthermore, pretreatment with the autophagy inhibitor chloroquine or 3-methyladenine showed the potential in attenuating the apoptosis rate induced by Sal B. Mechanistically, Sal B treatment inhibited the AKT/mTOR signaling cascade in vitro. Overexpression of AKT abolished the effects of Sal B on HCC cells, suggesting a critical role of the AKT/mTOR signaling pathway in Sal B-induced biological effects. Our results indicated that the mitochondrial pathway was involved in Sal B-induced apoptosis of HCC cells. Moreover, the AKT/mTOR signaling pathway was involved in Sal B-induced autophagy, which promoted apoptosis. This study may provide a promising
\end{abstract}

Correspondence to: Dr Weibing Zhang, Department of Pharmacy, The First Affiliated Hospital of Wenzhou Medical University, 2 NanBai Xiang, Wenzhou, Zhejiang 325000, P.R. China

E-mail: zhangweibing1991@163.com

Abbreviations: CQ, chloroquine; Cyto c, cytochrome $c$; HCC, hepatocellular carcinoma; LC3, microtubule-associated protein 1 light chain 3; PARP, poly(ADP-ribose) polymerase; PI, propidium iodide; PVDF, polyvinyldifluoride; SDS-PAGE, sodium dodecyl sulfate polyacrylamide gel electrophoresis; $\Delta \Psi \mathrm{m}$, membrane potential; 3-MA, 3-methyladenine

Key words: hepatocellular carcinoma, salvianolic acid B, AKT/mTOR signaling, apoptosis, autophagy strategy for using Sal B as a chemotherapeutic agent for patients with HCC.

\section{Introduction}

Hepatocellular carcinoma (HCC) is the fifth most common cancer in the world and the third cause of cancer mortality $(1,2)$. HCC is associated with infection with hepatitis B and C virus. Most HCC patients are diagnosed at the late stage and lose the opportunity for surgical operation (3). Conventional chemotherapy with oxaliplatin, doxorubicin, and fluorouracil as monotherapy or in combination for patients with advanced $\mathrm{HCC}$ is usually ineffective with a low response rate and severe side effects (4-6). Sorafenib, a multitargeted tyrosine kinase inhibitor which has been shown to improve by 2.8 months the median overall survival (OS) and by 2.7 months the median progression-free survival (PFS) for unresectable HCC (7). Currently, the prognosis of HCC is poor even with multidisciplinary comprehensive treatment. Therefore, identification and development of novel and safe treatment strategies are urgently needed to improve treatment outcomes.

In recent years, Chinese medicine is emerging as an attractive new generation of anticancer drugs due to their ability to effectively eliminate cancer cells with low toxicity (8-10). Salvia miltiorrhiza (Danshen), a popular Chinese herb, has been widely used for treating angina pectoris, myocardial infarction (MI) and stroke $(11,12)$. Salvianolic acid B (Sal B) is one of the major water-soluble compounds and active ingredients of Danshen $(13,14)$. Recently, the anticancer effects of Sal B have been demonstrated in human cancer cell lines including prostate, breast, liver, and head and neck squamous cell cancers (15-17). However, the effects of Sal B on HCC and the antitumor mechanisms have not been adequately studied.

Additionally, studies on the antitumor activity of Sal B have focused on inhibition of proliferation and induction of apoptosis (15). Sal B has also been reported to induce autophagy (18). Understanding the interplay between apoptosis and autophagy induced by Sal B in HCC may identify new targets for cancer therapy and improve the therapeutic efficiency of 
HCC. Currently, there are no reports on the effects of Sal B on the interplay between autophagy and apoptosis in HCC cells.

In this study, we demonstrated that Sal B inhibited the growth and induced cell death of human HCC cells in vitro. We also found that autophagy together with apoptosis is involved in Sal B-induced cell death in HCC cells. Inhibition of autophagy attenuated Sal B-induced cell death by reducing apoptosis. Moreover, Sal B-induced cell death was associated with AKT/mTOR signaling inhibition. These results suggest that Sal B could be a potential anticancer agent for the treatment of HCC.

\section{Materials and methods}

Chemicals and antibodies. Sal B was purchased from Sigma (Sigma-Aldrich Corp., St. Louis, MO, USA). 3-MA and CQ were purchased from J\&K Chemical Ltd. (J\&K Chemical Ltd., Beijing, China). JC-1 and LysoTracker Red were obtained from Invitrogen (Guangzhou, China). The primary antibodies against LC-3, p62, Beclin-1, cleaved PARP, cleaved caspase-3, cytochrome $c$, total or phospho-AKT (Ser473), total or phospho-mTOR (Ser2448), phospho-4EBP1 (Thr70), and phospho-P70S6K (Thr389) were purchased from Cell Signaling Technology (Boston, MA, USA). The secondary antibodies were HRP-conjugated anti-rabbit IgG purchased from Cell Signaling Technology. The FITC-conjugated antirabbit IgG was purchased from Beyotime (Beyotime, Nantong, China).

Cells lines and cultures. SK-Hep-1 and Bel-7404 cells were obtained from the American Type Culture Collection (ATCC, Rockville, MD, USA). The cell lines were frozen in liquid nitrogen soon after arrival. The experiments with these cells were carried out within 6 generations after resuscitation. SK-Hep-1 and Bel-7404 cells were cultured in Dulbecco's modified Eagle's medium (DMEM), containing $10 \%$ fetal bovine serum (FBS), $100 \mathrm{U}$ penicillin and $100 \mathrm{U}$ streptomycin at $37^{\circ} \mathrm{C}$ in a humidified incubator of $5 \% \mathrm{CO}_{2}$ and $95 \%$ air.

Cell viability assay. Cell proliferation was determined by MTS assay. Cells were seeded into 96-well plates and treated with Sal B, CQ, 3-MA or a combination. After treatment, $10 \mu 1$ MTS (Promega) was added into each well for 2-h incubation. The absorbance was measured using a model ELX800 Microplate Reader (Bio-Tek Instruments, Inc., Winooski, VT, USA) at $490 \mathrm{~nm}$ to calculate the proliferation. Three independent experiments were performed to determine the half maximal inhibitory concentration values $\left(\mathrm{IC}_{50}\right)$.

Colony forming assay. Cells were incubated at a density of 1,000 cells per well in 6 -well plates and treated with a determined dose of Sal B or vehicle control for 2 weeks. After fixation with $4 \%$ paraformaldehyde, the colonies formed were counterstained with crystal violet staining solution.

Apoptosis analysis. The cell apoptotic rate was determined by flow cytometry analysis using a fluorescein isothiocyanate (FITC) Annexin V Apoptosis Detection kit (KeyGen Biotech, Nanjing, China). Cells were collected by trypsinization, washed twice and resuspended in $1 \mathrm{X}$ binding buffer at a concentration of $1 \times 10^{6}$ cells $/ \mathrm{ml}$. Then, $100 \mu \mathrm{l}$ of cells were mixed with $5 \mu \mathrm{l}$ of FITC Annexin V and $5 \mu \mathrm{l}$ PI and incubated for $15 \mathrm{~min}$. The samples were sent out for analysis by flow cytometry. The results were analyzed with the BD FACSCalibur ${ }^{\mathrm{TM}}$ system.

Immunofluorescence analysis. Cells, seeded at $3 \times 10^{5}$ into 6 -well culture plates, were treated with a determined dose of Sal B for indicated intervals and were incubated with Lyso Tracker for $60 \mathrm{~min}$. Thereafter, cells were washed twice with PBS, followed by fixation in $4 \%$ paraformaldehyde and permeabilized with $1 \%$ CHAPS buffer $(150 \mathrm{mM} \mathrm{NaCl}, 10 \mathrm{mM}$ HEPES, $1.0 \%$ CHAPS) at room temperature for $15 \mathrm{~min}$. Hereafter, cells were incubated with anti-LC3 antibodies for $2 \mathrm{~h}$ at $37^{\circ} \mathrm{C}$, and incubated with FITC-conjugated anti-rabbit IgG for $1 \mathrm{~h}$ at $37^{\circ} \mathrm{C}$, and the cell nuclei were stained by DAPI (Invitrogen) for $15 \mathrm{~min}$. Samples were examined under a Zeiss LSM 710 fluorescence microscopy system (Carl Zeiss Inc, Dublin, CA, USA). Images were processed with ZEN LE software. For quantification of LC3-positive cells, 150-200 cells were randomly selected from the acquired images and counted. Cells with more than five dots of specific green or yellow signals were considered to be LC3-positive.

Transmission electron microscope. Cells, seeded at $3 \times 10^{5}$ into 6-well culture plates, were treated with a dose of $200 \mu \mathrm{M}$ Sal B for $24 \mathrm{~h}$. Then, the cells were washed and fixed for $30 \mathrm{~min}$ in $2.5 \%$ glutaraldehyde. The samples were treated with $1.5 \%$ osmium tetroxide, dehydrated with acetone and embedded in Durcupan resin. Thin sections were stained with lead citrate and examined by TECNAI 10 electron microscopy (Philips, Eindhoven, The Netherlands) at $60 \mathrm{kV}$.

RNA interference. For ATG6 (Beclin-1) interference, two siRNA oligonucleotides targeting ATG6 were synthesized by GenePharma (Shanghai, China). The sequences of the sense strands of the RNAs targeting ATG6 used in this study were as follows: ATG6 siRNA-1 (si1): 5'-GUGAGAAGCAAGCCCU UAUTT-3', ATG6 siRNA-2 (si2): 5'-CUCCAGUGCUAAGCU ACAU-TT-3'. A non-specific oligo that is not complementary to any human genes was used as a negative control. The mixture of sil and si2 was used to increase the inhibitory activity. Cells were transfected with siRNA using HiPerFect (Qiagen) according to the manufacturer's protocol.

Plasmids transfection. The pcDNA3-AKT-T7 plasmid was a gift from William Sellers (Addgene plasmid 9003). Cells were seeded into 6-well plates the day before transfection. Attractene (Qiagen) was used for transfection according to the manufacturer's protocol. Ater $24 \mathrm{~h}$ of incubation, the cells were subjected to different treatments. pcDNA3 empty vectors were used as controls for transfection experiments.

Isolation of cytosolic protein fractions. Cells were seeded in 6-well culture plates at $40-50 \%$ confluence. The next day, the cells were incubated with a determined dose of Sal B. Then, the cells were trypsinized, washed twice with ice-cold PBS, and the cytosolic protein fractions were isolated using the Cell Mitochondria Isolation kit (Beyotime) according to the manufacturer's protocol. 
A

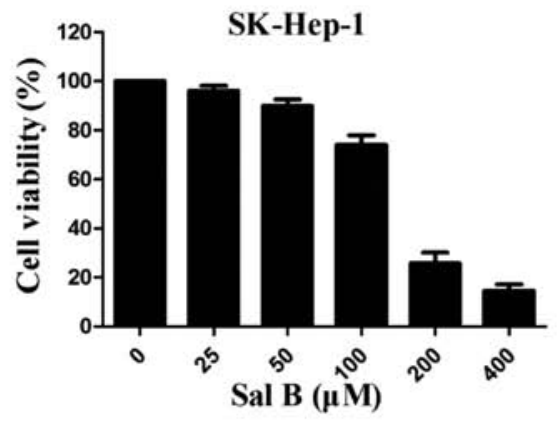

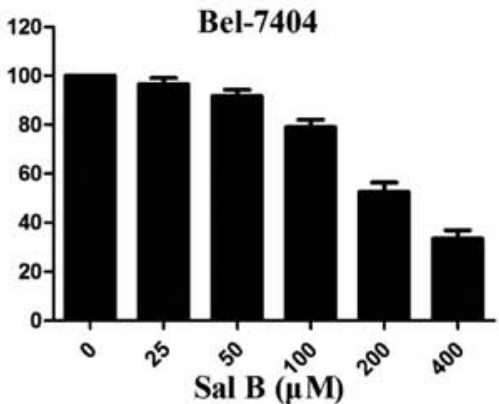

200

B Sal B $(\mu \mathrm{M}) \quad 0$
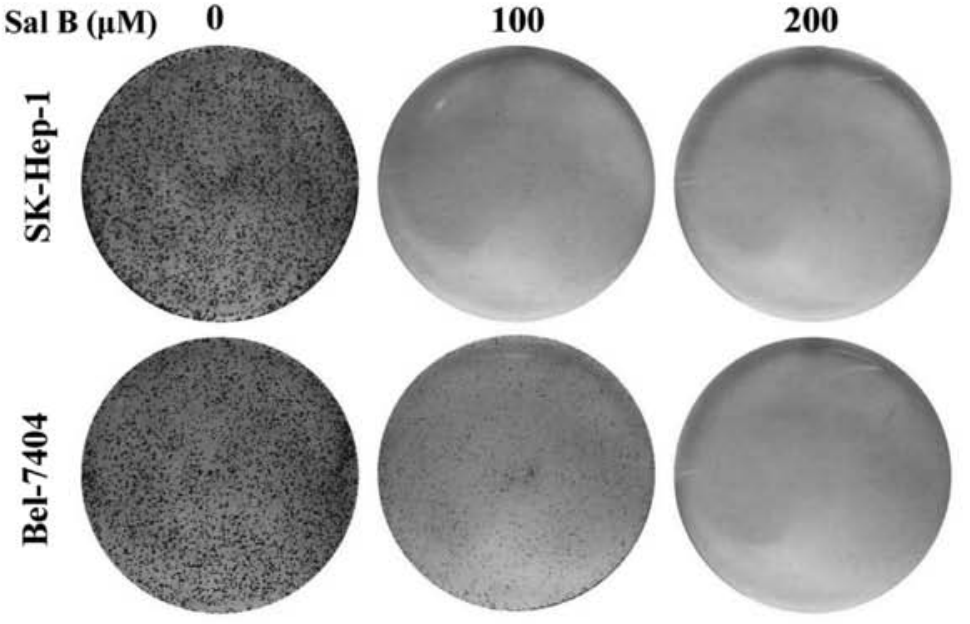

C

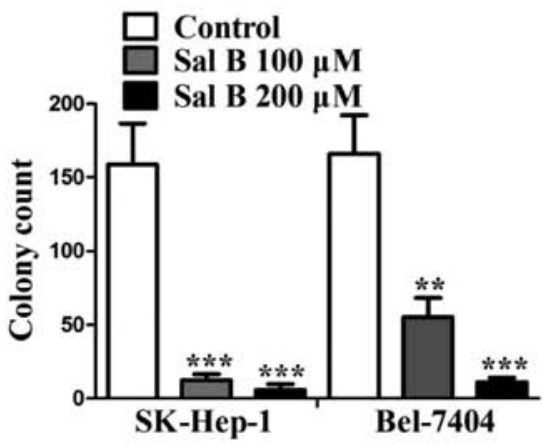

D

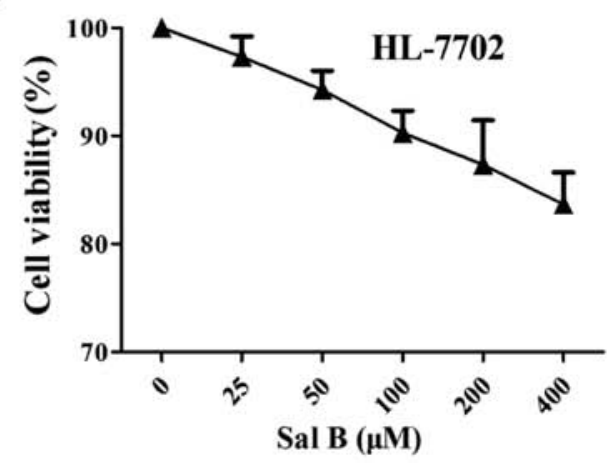

Figure 1. Sal B suppresses the growth of HCC cells. (A) Inhibitory effect of Sal B on SK-Hep-1 and Bel-7404 cells. The percentage of viable cells was measured by the MTS assay. (B) Sal B impairs the colony-forming ability of HCC cells in vitro. The control cells are significantly more efficient in forming colonies than the Sal B-treated cells. (C) The total number of colonies, each containing $>50$ cells, was determined. (D) Cytotoxicity of Sal B on normal human liver cells. After treatment with Sal B for $48 \mathrm{~h}$, the percentage of viable HL-7702 cells was measured by the MTS assay. ${ }^{* *} \mathrm{P}<0.01,{ }^{* * *} \mathrm{P}<0.001$. All data are representative of three independent experiments.

Western blot analysis. Western blot analysis was performed as described previously (19). Briely, proteins $(40 \mu \mathrm{g})$ were resolved by $6-15 \%$ sodium dodecyl sulfate-polyacrylamide gel electrophoresis, transferred to polyvinylidene diluoride membranes (Millipore, Billerica, MA, USA). The membranes were then blocked in $5 \%(\mathrm{w} / \mathrm{v})$ skimmed-milk for $1 \mathrm{~h}$, followed by incubation with appropriate primary antibodies (diluted 1:1,000-1:5,000) overnight at $4^{\circ} \mathrm{C}$. Bound antibodies were detected with peroxidase-conjugated goat anti-rabbit or goat anti-mouse secondary antibodies and the blots developed using enhanced chemiluminescence (Millipore). Western blot analysis data were quantified by ImageJ densitometric analysis and normalized by GAPDH.
Statistical analysis. All experiments were performed at least three times, and the data were presented as the mean \pm standard deviation. GraphPad Prism 5.0 was used for statistical analysis. A difference was considered significant at $\mathrm{P}<0.05$.

\section{Results}

Sal B inhibits growth of HCC cells. We first examined the inhibitory effect of Sal B on viability in HCC cell lines. As shown in Fig. 1A, Sal B significantly inhibited the growth of cancer cells in a dose-dependent manner, with a half maximal inhibitory concentration $\left(\mathrm{IC}_{50}\right)$ of $143.82 \mu \mathrm{M}$ for SK-Hep-1 and $240.11 \mu \mathrm{M}$ for Bel-7404 after $48 \mathrm{~h}$ of exposure. 
A

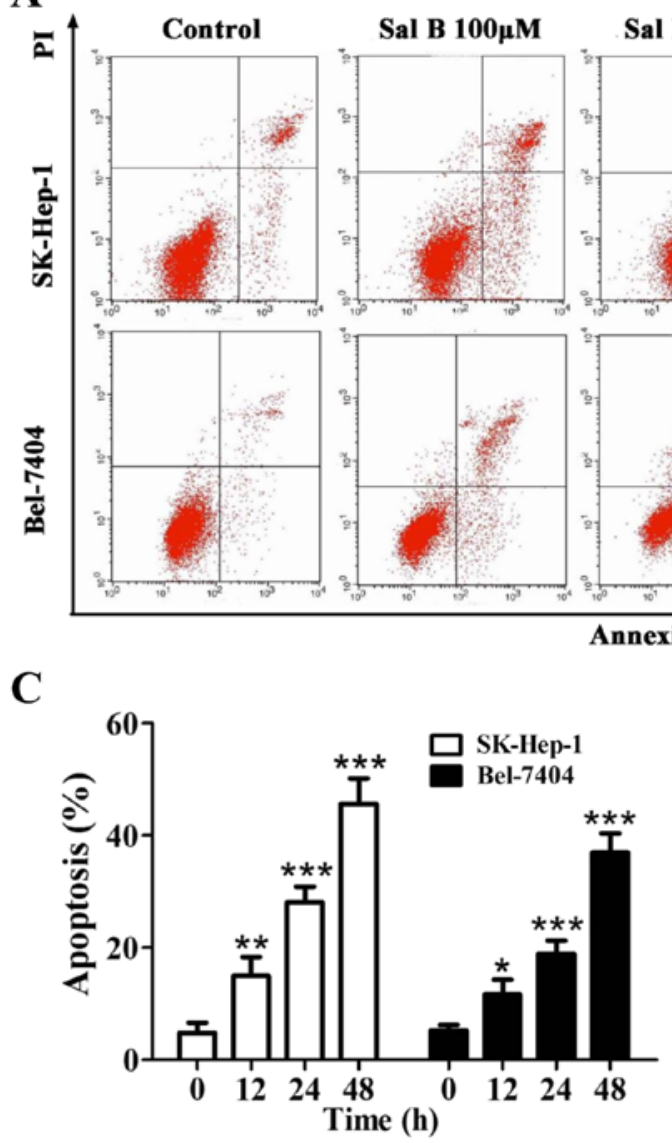

B

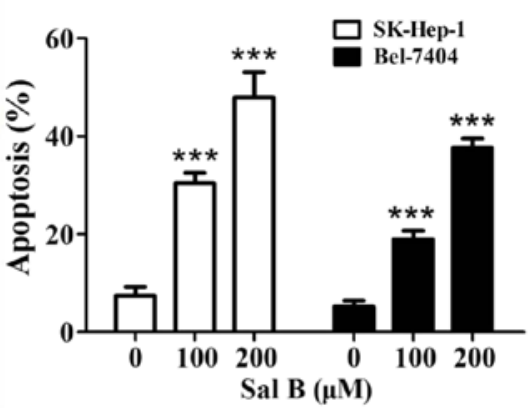

D

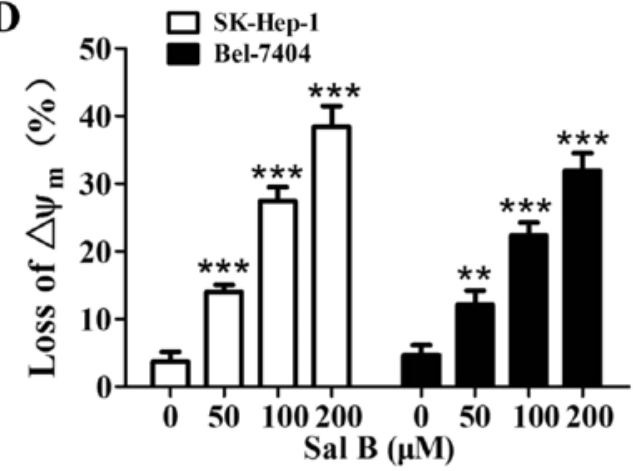

$\mathbf{E}$

Bel-7404

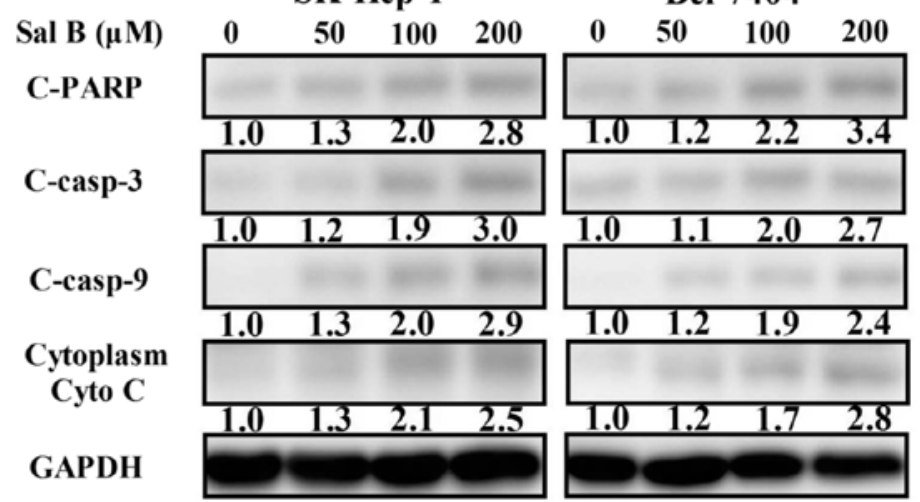

Figure 2. Sal B induces apoptosis in HCC cells. Cells were treated with Sal B for $48 \mathrm{~h}$ before staining with Annexin V (AV) and propidium iodide (PI) (A), and the apoptotic rates were determined by flow cytometry (B). (C) Cells were treated with $200 \mu \mathrm{M}$ Sal B for the indicated intervals before staining with AV and PI, and the apoptotic rates were determined by flow cytometry. (D) Cells were treated with Sal B for $48 \mathrm{~h}$ before staining with JC-1, and the mitochondrial membrane potential was evaluated by flow cytometry. (E) The expression of apoptosis-associated proteins in cells treated with Sal B for $24 \mathrm{~h}$. Relative quantity of proteins were calculated by ImageJ densitometric analysis and normalized by GAPDH. ${ }^{*} \mathrm{P}<0.05,{ }^{* *} \mathrm{P}<0.01,{ }^{* * * *} \mathrm{P}<0.001$. The data are representative of three independent experiments.

Colony-formation experiments also indicated that Sal B markedly inhibited the growth of HCC cells (Fig. 1B and C). We also investigated the effects of Sal B on the growth of HL-7702 cells (a normal human liver cell line). The results showed that the cytotoxic effect of Sal B on HL-7702 cells $\left(\mathrm{IC}_{50} 758.63 \mu \mathrm{M}\right)$ appeared much lower than that observed in SK-Hep-1 and Bel-7404 cells (Fig. 1D).

Sal B induces apoptotic cell death in HCC cells. To examine the cell growth inhibition induced by Sal B related to apoptosis,
Sal B-treated cells were stained with propidium iodide (PI)/ Annexin V-FITC and quantified by flow cytometry. The quantification shown for apoptosis reflected the values for total apoptosis. We also determined whether apoptosis was doseor time-dependent altered in cells treated with Sal B. Sal B induced a dose- and time-dependent increase of apoptosis in HCC cells (Fig. 2A-C).

Then we measured the mitochondrial membrane potential $(\Delta \Psi \mathrm{m})$ by flow cytometry and found that Sal B treatment led to depolarization of $\Delta \Psi_{\mathrm{m}}$ in a dose-dependent manner (Fig. 2D). 

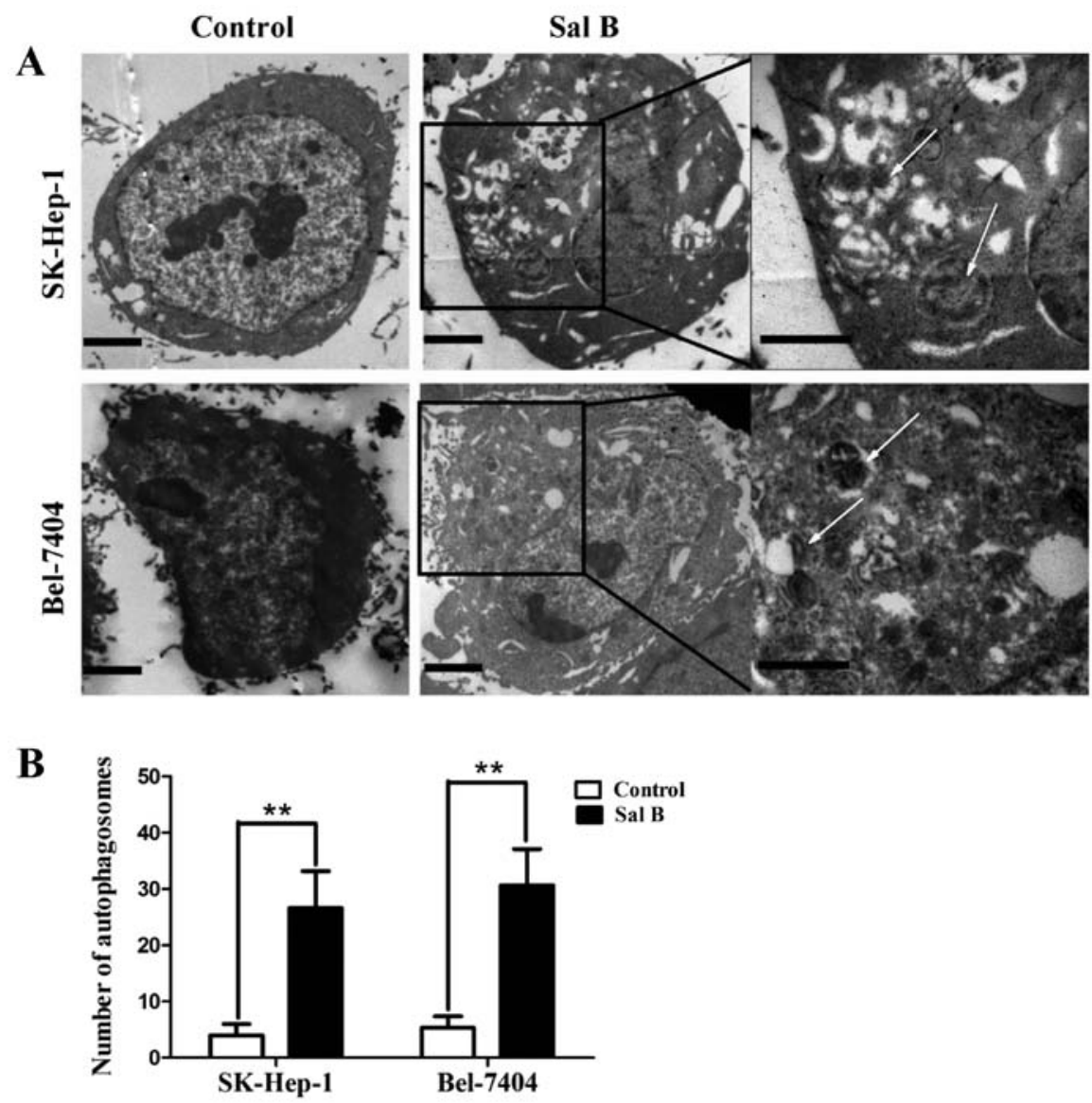

Figure 3. TEM depicting ultrastructures of autophagosome in HCC cells treated with Sal B. (A) Numerous autophagosomes appeared in cells treated with $200 \mu \mathrm{M}$ Sal B for $24 \mathrm{~h}$. Scale bar, $1 \mu \mathrm{m}$. (B) The number of autophagosomes was calculated by continuous counts within 10 fields under high resolution. The data are representative of three independent experiments.

Western blot analyses showed that cleaved caspase-9, cleaved caspase-3, cleaved poly(ADP-ribose) polymerase (PARP), and cytosolic Cyto c were increased after treatment with Sal B for $24 \mathrm{~h}$ (Fig. 2E). The level of cleaved PARP, cleaved caspase-3, cleaved caspase-9, and cytosolic Cyto c increased 2.8-, 3.0-, 2.9- and 2.5-fold, respectively, in SK-Hep-1 cells treated with $200 \mu \mathrm{M}$ Sal B for $24 \mathrm{~h}$ (Fig. 2E). The level of cleaved PARP, cleaved caspase-3, cleaved caspase-9, and cytosolic Cyto c increased 3.4-, 2.7-, 2.4- and 2.8-fold, respectively, in Bel-7404 cells treated with $200 \mu \mathrm{M} \mathrm{Sal} \mathrm{B}$ for $24 \mathrm{~h}$ (Fig. 2E). These results demonstrated that Sal B induced apoptosis in the HCC cells.

Sal B induces autophagy in HCC cells. Apoptosis and autophagy are highly interactive. To examine whether Sal B could induce autophagy, the treated cells were analyzed by western blot analysis and electron microscopy. The characteristics of autophagosomes are described as double-layer structure with cytoplasmic components. After treatment with Sal B for $24 \mathrm{~h}$, most of the HCC cells displayed an extensive accumulation of double structures with a broad range of morphologies, indicating the formation of autophagosomes (Fig. 3). The significantly increased expression of LC3-II, Beclin-1 and the attenuated expression of p62/SQSTM1 were observed in cells treat with Sal B for $24 \mathrm{~h}$ (Fig. 4A). The level of LC3-II and Beclin-1 increased 3.1- and 2.3-fold, while the level of p62 decreased $80 \%$, in SK-Hep-1 cells treated with
$200 \mu \mathrm{M} \mathrm{Sal} \mathrm{B}$ for $24 \mathrm{~h}$ (Fig. 4A). The level of LC3-II and Beclin-1 increased 3.5- and 2.6-fold, respectively, while the level of p62 decreased $70 \%$, in Bel-7404 cells treated with $200 \mu \mathrm{M} \mathrm{Sal} \mathrm{B}$ for $24 \mathrm{~h}$ (Fig. 4A).

For further confirmation, the cells were incubated with an antibody against microtubule-associated protein 1 light chain 3 (LC3). The punctate LC3 II-labeled autophagolysosome vacuoles were frequently observed in cells treated with Sal B for $48 \mathrm{~h}$ compared with their controls (Fig. 4B). Next, we determined whether autophagy was time-dependently altered in cells treated with Sal B. A time-dependent increase of punctate LC3-II dots was observed from 12-, 24- and 48-h in cells treated with Sal B (Fig. 4C).

Interactions between Sal B-induced autophagy and apoptotic cell death in HCC cells. The relationships between autophagy and apoptosis are complicated. There is consensus that the relationships between autophagy and apoptosis are highly depended on the tumor types and stimulus characteristic. To confirm the role of Sal B-induced autophagy in HCC cells promoted or inhibited apoptosis, we treated the cells with the autophagy inhibitor 3-MA and CQ. Addition of $5 \mathrm{mM} 3-\mathrm{MA}$ or $5 \mu \mathrm{M} \mathrm{CQ}$ attenuated Sal B-induced apoptosis in HCC cells (Fig. 5A). In addition, siRNAs against Beclin-1 was used to block autophagy. The level of Beclin-1 decreased $70 \%$ in SK-Hep-1 cells and 60\% in Bel-7404 cells treated with siRNAs 
A

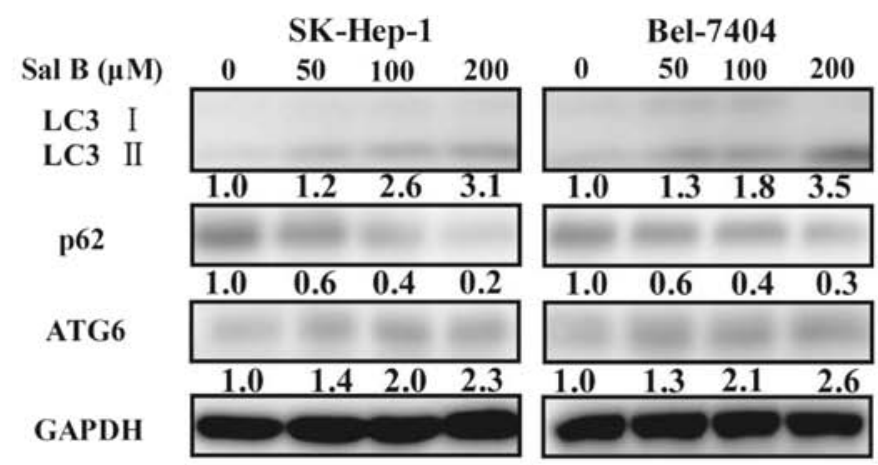

B

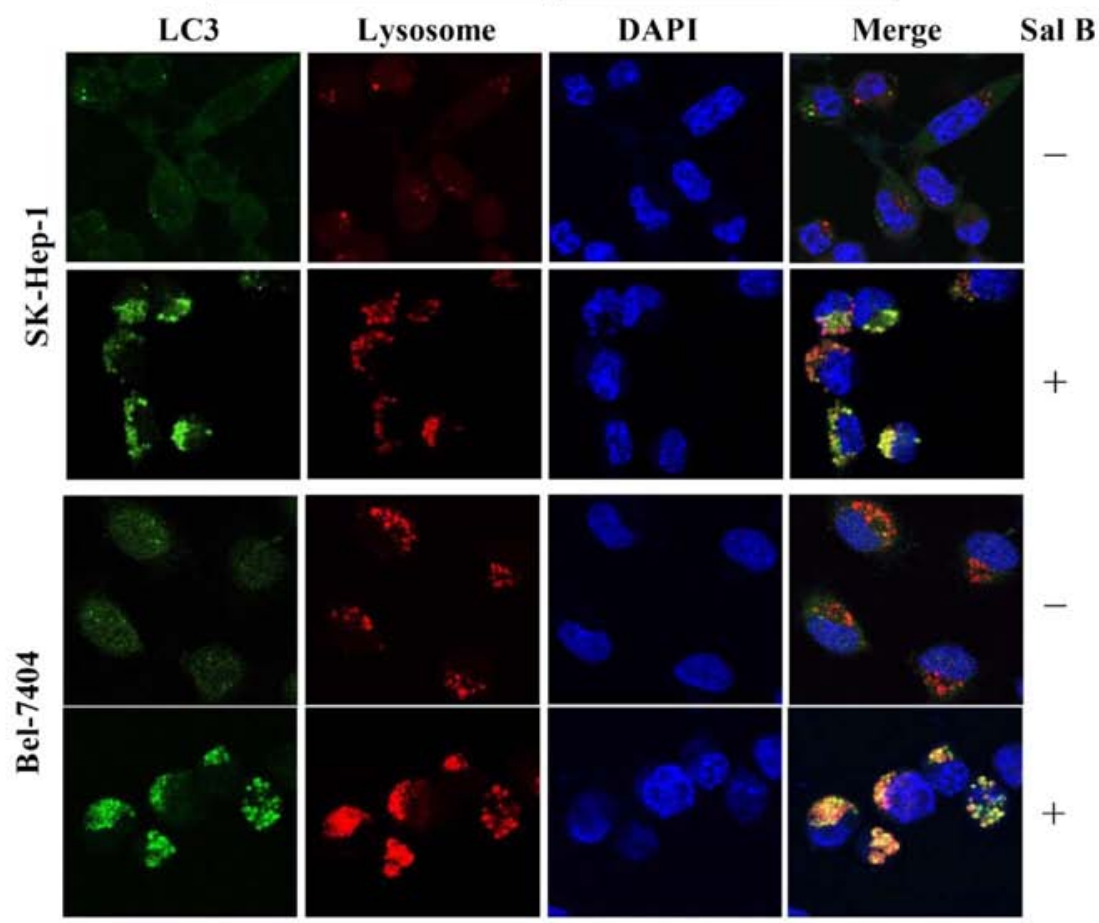

C

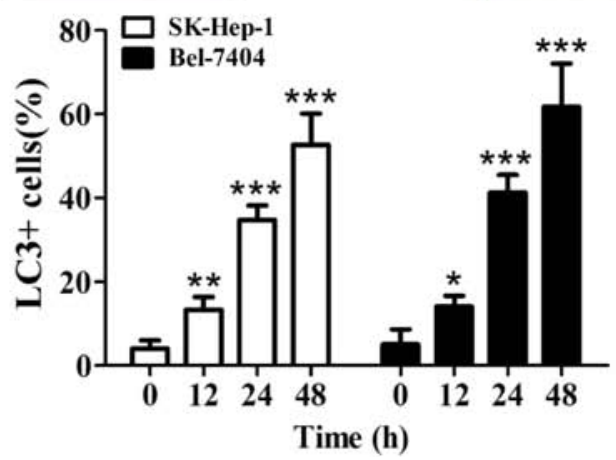

Figure 4. Effect of Sal B on LC3 processing. (A) Immunoblotting for p62, Beclin-1, and LC3 using lysates from cells treated with Sal B for $24 \mathrm{~h}$. Relative quantity of proteins were calculated by ImageJ densitometric analysis and normalized by GAPDH. (B) Cells were treated with DMSO or $200 \mu \mathrm{M}$ Sal B for $48 \mathrm{~h}$ before they were labeled with fluorescence and imaged by fluorescence microscope. Green, FITC-labeled LC3. Red, LysoTracker-labeled lysosome. Blue, DAPI-labeled nuclei. (C) Cells were treated with DMSO or $200 \mu \mathrm{M}$ Sal B for the indicated intervals before they were labeled with fluorescence and imaged by fluorescence microscope. The percentage of puncta-positive cells was quantified by automated image acquisition and analysis using a threshold of $>5$ dots/cell. ${ }_{* * * *} \mathrm{P}<0.001$. The data are representative of three independent experiments.

against Beclin-1 (Fig. 5B). Inhibition of autophagy attenuated Sal B-induced apoptosis in HCC cells (Fig. 5B).

Western blot analysis was used to detect the influence of 3-MA pretreatment on cleaved PARP and cleaved caspase-3 expression induced by Sal B. Autophagy level was found to significantly decrease after 3-MA pretreatment, and the expressions of cleaved PARP and cleaved caspase- 3 also decreased
(Fig. 5C). The level of cleaved PARP, cleaved caspase-3, and LC3-II decreased from 4.5- to 2.1-, 2.7- to 1.9- and 5.5- to 1.6-fold, respectively, in SK-Hep-1 cells treated with $200 \mu \mathrm{M}$ Sal B and the combination of Sal B and 3-MA for $24 \mathrm{~h}$ (Fig. 5C). The level of cleaved PARP, cleaved caspase-3, and LC3-II decreased from 3.4- to 2.5-, 3.1- to 2.2- and 4.2-to 1.2-fold, respectively, in Bel-7404 cells treated with $200 \mu \mathrm{M}$ Sal B and 
A
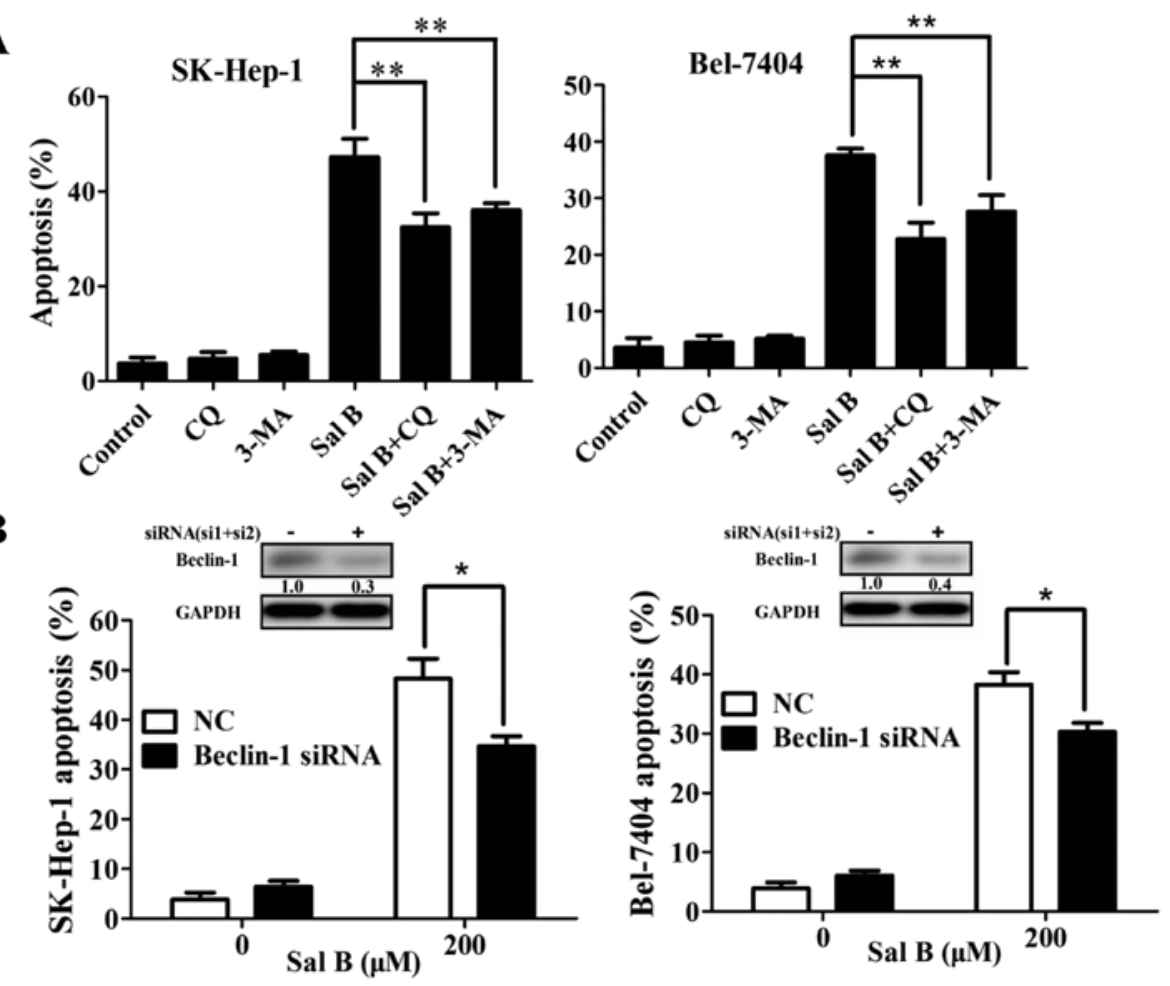

C

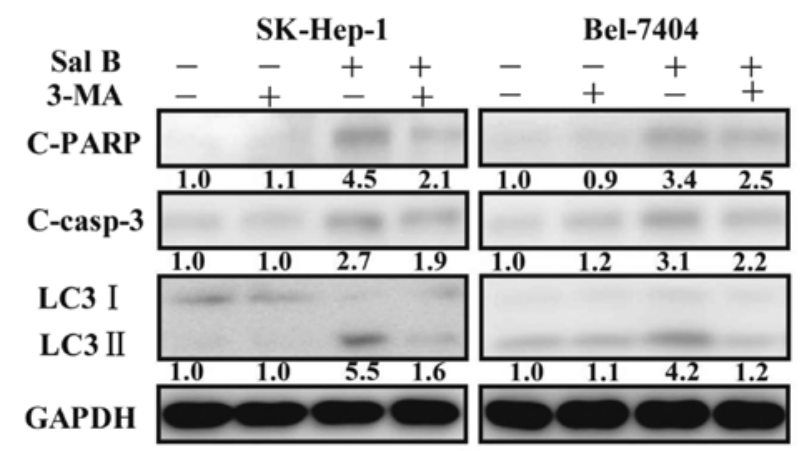

Figure 5. Inhibition of autophagy attenuates Sal B-induced apoptosis in HCC cells. (A) Cells were treated with $200 \mu \mathrm{M}$ Sal B in the presence or absence of $\mathrm{CQ}(5 \mu \mathrm{M})$ or 3-MA $(5 \mathrm{mM})$ for $48 \mathrm{~h}$. Apoptotic rate was measured by flow cytometry. (B) Cells were transfected with siRNA against Beclin-1 for $24 \mathrm{~h}$, then treated with Sal B for $48 \mathrm{~h}$. Apoptotic rate was measured by flow cytometry. (C) Immunoblotting for PARP, cleaved caspase-3 and LC 3 using lysates from cells treated with $200 \mu \mathrm{M} \mathrm{Sal} \mathrm{B}$ in the presence or absence of 3-MA. Relative quantity of proteins were calculated by ImageJ densitometric analysis and normalized by GAPDH. ${ }^{*} \mathrm{P}<0.05,{ }^{* * *} \mathrm{P}<0.01$. The data are representative of three independent experiments.

the combination of Sal B and 3-MA for $24 \mathrm{~h}$ (Fig. 5C). All the results demonstrated that the inhibition of autophagy in HCC cells had the potential of attenuating Sal B-induced apoptosis.

AKT/mTOR signaling pathway is involved in Sal B-induced autophagy in HCC cells. AKT/mTOR signaling pathway is the key regulatory molecule of both autophagy and apoptosis. Therefore, we investigated whether the AKT/mTOR pathway played a central role in Sal B-mediated cell death. Western blot analysis confirmed that the levels of phosphorylated AKT, mTOR and its downstream effector p70S6K and p-4EBP1 were significantly reduced by Sal B (Fig. 6A). The level of phosphorylated AKT, mTOR, p70S6K and p-4EBP1 decreased 70, 70,70 and $60 \%$, respectively, in SK-Hep-1 cells treated with $200 \mu \mathrm{M}$ Sal B for $24 \mathrm{~h}$ (Fig. 6A). The level of phosphorylated AKT, mTOR, p70S6K and p-4EBP1 decreased 70, 80, 70 and $70 \%$, respectively, in Bel-7404 cells treated with $200 \mu \mathrm{M}$ Sal B for $24 \mathrm{~h}$ (Fig. 6A).
To further identify the role of AKT in Sal B-induced biological effects, HCC cells were transiently transfected with pcDNA3-AKT-T7 plasmid. The level of phosphorylated AKT and total AKT increased 2.0- and 5.5-fold in SK-Hep-1 cells, while 1.6- and 5.2-fold in Bel-7404 cells transfected with pcDNA3-AKT-T7 plasmid (Fig. 6D). As shown in Fig. 6B-D, enforced expression of AKT significantly attenuated Sal B-induced growth inhibition, and apoptosis. Furthermore, Sal B-induced autophagy was also decreased in the transfected cells (Figs. 6D and 7). The level of cleaved PARP and LC3-II decreased from 2.6- to 1.4- and 2.2- to 1.3-fold, respectively, in SK-Hep-1 cells treated with $200 \mu \mathrm{M}$ Sal B and the combination of Sal B and pcDNA3-AKT (Fig. 6D). The level of cleaved PARP and LC3-II decreased from 2.7- to 1.5- and 1.8- to 1.2-fold, respectively, in Bel-7404 cells treated with $200 \mu \mathrm{M}$ Sal B and the combination of Sal B and pcDNA3-AKT (Fig. 6D). These results suggested that overexpression of AKT can override the Sal B-induced 
A

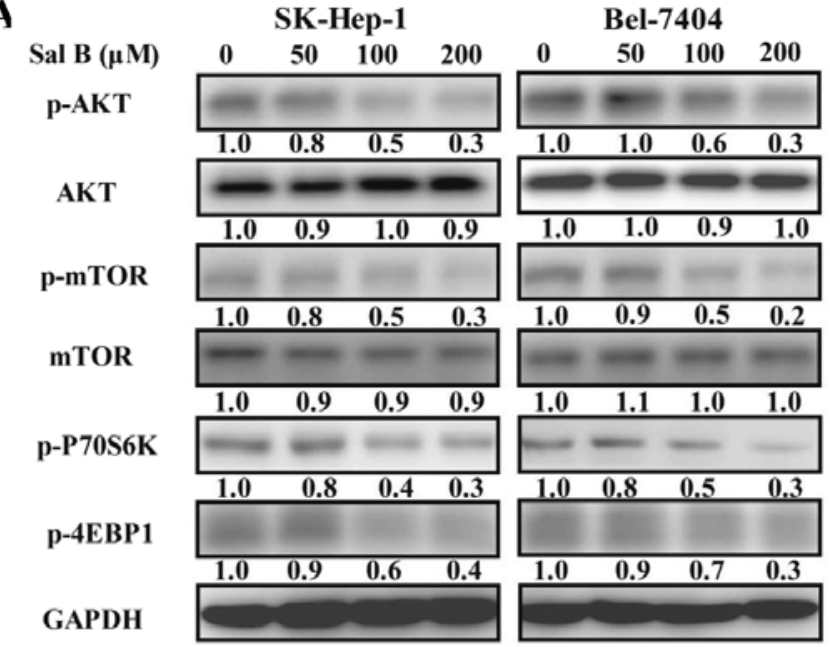

C

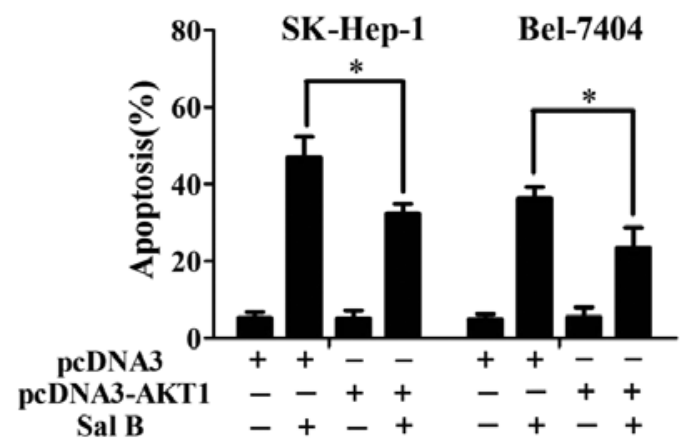

B
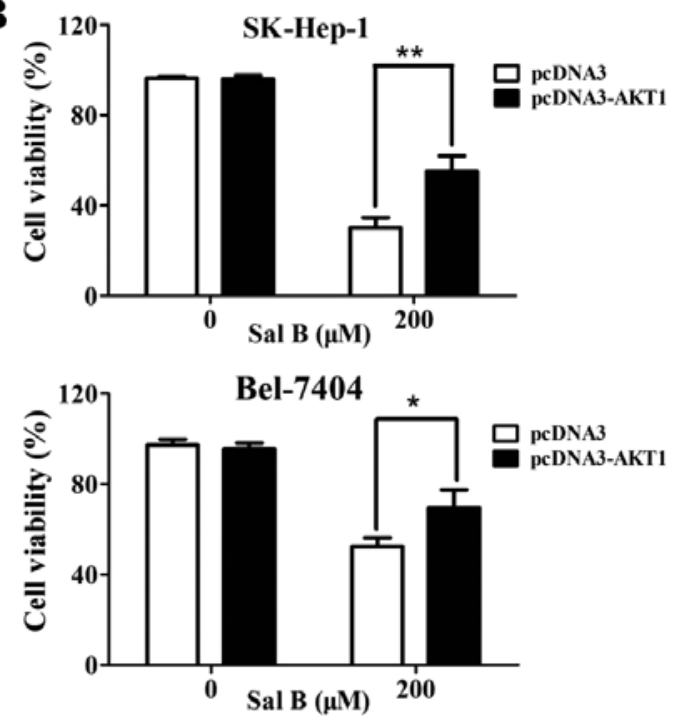

D

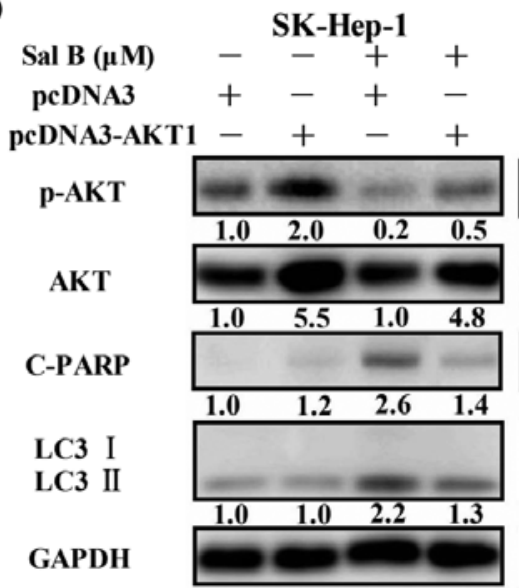

Bel-7404

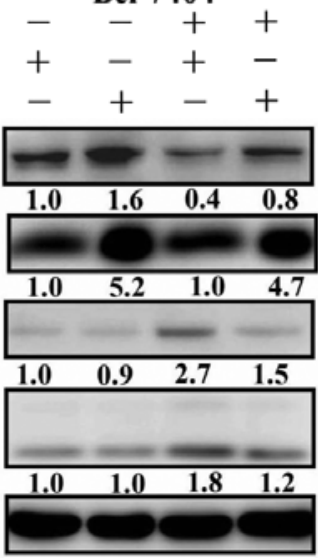

Figure 6. AKT signaling is a critical mediator in regulating Sal B-mediated biological effects. (A) Immunoblotting for phospho-AKT, mTOR, S6K, 4EBP1, total AKT, and mTOR in cells treated with Sal B for $24 \mathrm{~h}$. Relative quantity of proteins was calculated by ImageJ densitometric analysis and normalized by GAPDH. After transient overexpression of AKT, cells were treated with Sal B, and then cell viability (B) and apoptotic rate (C) were determined by MTS assay or flow cytometry. (D) Autophagy and apoptosis-associated proteins, including LC3, cleaved PARP, and total AKT were analyzed by immunoblotting. Relative quantity of proteins were calculated by ImageJ densitometric analysis and normalized by GAPDH. ${ }^{*} \mathrm{P}<0.05$, $^{* *} \mathrm{P}<0.01$. The data are representative of three independent experiments.

biological effects in HCC cells. Taken together, these data demonstrate that $\mathrm{AKT}$ is a critical mediator in regulating Sal B-mediated biological effects.

\section{Discussion}

Currently, the prognosis of HCC is poor even with multidisciplinary comprehensive treatment, and recurrence rate is $>50 \%(20,21)$. There is an urgent need for development of efficacious therapies. Growing evidence indicates that Chinese medicine plays a promising role in developing novel anticancer drugs. Understanding the anti-neoplastic mechanisms of Chinese medicine may help improve the efficacy of these agents.

In this study, we demonstrated that Sal B markedly inhibited the proliferation of HCC cells. We also showed that Sal B induced mitochondria-mediated apoptosis in HCC cells, accompanied by a decrease of mitochondrial potential, and increase of cytosol cytochrome $c$. Release of cytochrome $c$ from the mitochondria into cytosol activates intrinsic apoptosis (22). Cytosolic Cyto c initiates the apoptotic process by activating a downstream cascade of caspases through processing of procaspase-9 (23). In accord with our findings, some researchers reported that Sal B inhibited the growth of cancer cells through induction of apoptosis $(24,25)$.

Both apoptosis and autophagy are crucial mechanisms regulating cell survival (26). We thus examined whether Sal B induced autophagy. Autophagy is a tightly regulated intracellular self-digestive process involving the lysosomal degradation of cytoplasmic organelles and proteins $(27,28)$. In this process, cells digest their own cellular contents by lysosomal degradation and recycle the ingredients to maintain cell survival (29-31). Our data revealed that Sal B could induce autophagy accompanied by apoptosis in HCC cells. Apoptosis and autophagy could be induced by the same stimulus, but the interaction between them was still unclear. In our study, we found that suppression of autophagy by pharmacological inhibitors (3-MA and CQ) or Beclin-1 siRNA 
$\mathbf{A}$

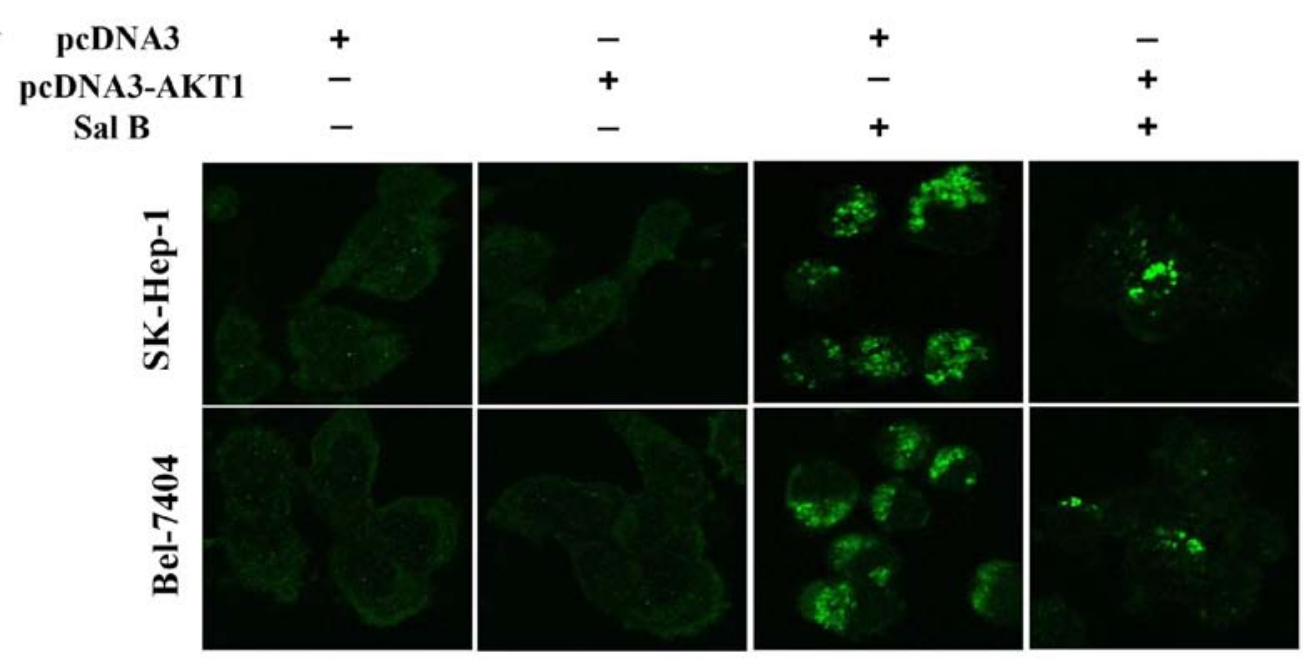

B

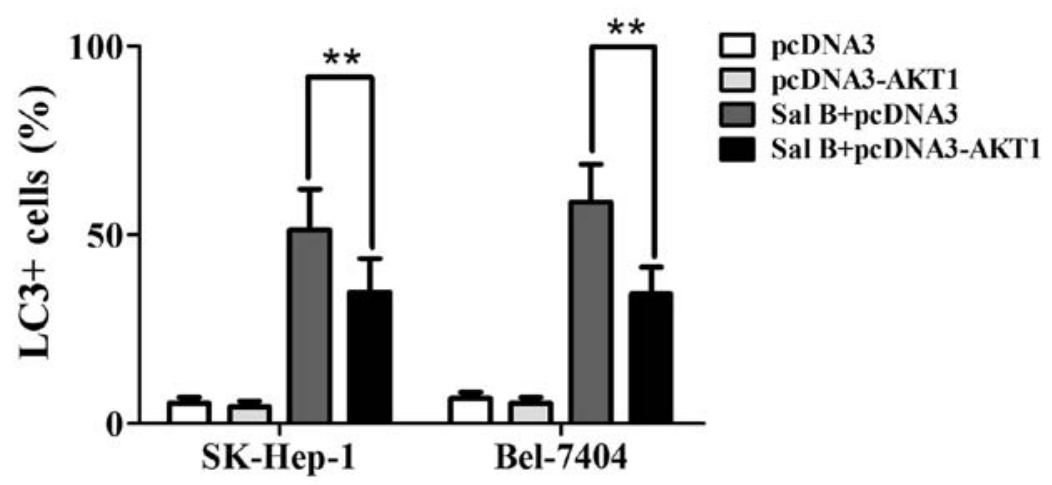

Figure 7. Overexpression of AKT inhibits Sal B-induced autophagy. (A) After transient transfection of AKT expression plasmid, cells were treated with $200 \mu \mathrm{M}$ Sal B for $24 \mathrm{~h}$. Then, the cells were labeled with fluorescence and imaged by fluorescence microscopy. Green, FITC-labeled LC3. (B) The percentage of puncta-positive cells was quantified by automated image acquisition and analysis using a threshold of $>5$ dots/cell. ${ }^{* *} \mathrm{P}<0.01$. The data are representative of three independent experiments.

decreased Sal B-induced apoptosis in HCC cells, revealing that the autophagy induced by Sal B promoted HCC cell apoptosis. These results were consistent with the findings of Kim et al (32), who reported that the inhibition of autophagy decreased docosahexaenoic acid-induced apoptosis in nonsmall cell lung cancer cells, indicating that autophagy was a prerequisite for apoptotic cell death. Autophagy can inhibit, delay or promote apoptosis (33-35). The mechansims of autophagy promoting apoptosis may include upregulation of cells susceptible to drug-induced apoptosis and activating of caspases $(36,37)$. In this study, our data showed that apoptosis level was significantly increased with upregulation of autophagy level. We hypothesize that Sal B could trigger apoptosis and autophagy simultaneously, whereas autophagy increased HCC cells susceptible to Sal B-induced apoptosis. Inhibition of autophagy could significantly reduce the extent of Sal B-induced apoptosis. The interaction between autophagy and apoptosis is extremely complex and needs further investigation.

The molecular mechanism mediating apoptosis and autophagy are complicated. Increasing evidence indicates that autophagy and apoptosis share many common regulatory molecules, such as AKT/mTOR signaling pathway (38). It is well known that the AKT/mTOR pathway plays an important role in cell growth, survival, differentiation and metabolism (39). The aberrant activation of AKT/mTOR signaling pathway contributes to a poor prognosis and plays a critical role in carcinogenesis of HCC (40). Inhibition of AKT/mTOR signaling pathway causes cell death associated with apoptosis and autophagy (41). The downstream target of Akt/mTOR pathway can potently block Bad-induced apoptosis by phosphorylation of Bad at S136 site to disrupt Bad's binding to $\mathrm{Bcl}-\mathrm{XL}$ and/or Bcl-2. Thus, inhibition of Akt/mTOR pathway possibly increases apoptosis (42).

Our results show that Sal B treatment decreases AKT/mTOR pathway activity. This inhibitory effect was correlated with the decrease of phosphorylation of AKT, mTOR and their downstream targets, p70S6K and 4E-BP1. Overexpression of AKT abolished the effects of Sal B on HCC cells. These findings indicate that Sal B induces apoptosis and autophagy in HCC cells through inhibition of the AKT/mTOR signaling pathway. Moreover, the appearance of apoptosis and autophagy after Sal B treatment is closely linked to the inhibition of AKT/mTOR pathway, demonstrating this pathway plays a pivotal role in HCC treatment.

In conclusion, our results demonstrate for the first time that Sal B suppressed cell proliferation and induces autophagy and apoptosis in HCC cells through the AKT/mTOR pathway. 
Sal B could act as a new anticancer agent for HCC by inducing apoptosis and autophagy. These results will expand our knowledge of the anticancer molecular mechanisms of Sal B and the interaction between autophagy and apoptosis.

\section{Acknowledgements}

This study was supported by the grants from Medical Health Research Project of Zhejiang province (2015KYB301); Science and Technology Planning Project of Hangzhou (20150733Q20); Health Science and Technology Plan Projects of Hangzhou (2014A11); Science and Technology Planning Project of Wenzhou (2015Y0395).

\section{References}

1. Fares $\mathrm{N}$ and Peron JM: Epidemiology, natural history, and risk factors of hepatocellular carcinoma. Rev Prat 63: 216-217, 220-212, 2013 (In French).

2. Torre LA, Bray F, Siegel RL, Ferlay J, Lortet-Tieulent J and Jemal A: Global cancer statistics, 2012. CA Cancer J Clin 65 87-108, 2015 .

3. Karaman B, Battal B, Sari S and Verim S: Hepatocellular carcinoma review: Current treatment, and evidence-based medicine. World J Gastroenterol 20: 18059-18060, 2014.

4. Uhm JE, Park JO, Lee J, Park YS, Park SH, Yoo BC, Paik SW, Koh KC, Kang WK and Lim HY: A phase II study of oxaliplatin in combination with doxorubicin as first-line systemic chemotherapy in patients with inoperable hepatocellular carcinoma. Cancer Chemother Pharmacol 63: 929-935, 2009.

5. Ge S and Huang D: Systemic therapies for hepatocellular carcinoma. Drug Discov Ther 9: 352-362, 2015.

6. Yoon EL, Yeon JE, Lee HJ, Suh SJ, Lee SJ, Kang SH, Kang K, Yoo YJ, Kim JH, Yim HJ, et al: Systemic cytotoxic chemotherapy of patients with advanced hepatocellular carcinoma in the era of sorafenib nonavailability. J Clin Gastroenterol 48: e22-e29, 2014.

7. Llovet JM, Ricci S, Mazzaferro V, Hilgard P, Gane E, Blanc JF, de Oliveira AC, Santoro A, Raoul JL, Forner A, et al; SHARP Investigators Study Group: Sorafenib in advanced hepatocellular carcinoma. N Engl J Med 359: 378-390, 2008.

8. Han LT, Fang Y, Cao Y, Wu FH, Liu E, Mo GY and Huang F: Triterpenoid saponin flaccidoside II from Anemone flaccida triggers apoptosis of NF1-associated malignant peripheral nerve sheath tumors via the MAPK-HO-1 pathway. Onco Targets Ther 9: 1969-1979, 2016.

9. Xu J, Song Z, Guo Q and Li J: Synergistic effect and molecular mechanisms of traditional Chinese medicine on regulating tumor microenvironment and cancer cells. BioMed Res Int 2016 1490738,2016

10. Feng M, Zhong LX, Zhan ZY, Huang ZH and Xiong JP Resveratrol treatment inhibits proliferation of and induces apoptosis in human colon cancer cells. Med Sci Monit 22: 1101-1108, 2016.

11. Kim MS, Bang JH, Lee J, Kim HW, Sung SH, Han JS and Jeon WK: Salvia miltiorrhiza extract protects white matter and the hippocampus from damage induced by chronic cerebral hypoperfusion in rats. BMC Complement Altern Med 15: 415, 2015.

12. Jiang Y, Wang L, Zhang L, Wang T, Zhou Y, Ding C, Yang R, Wang $X$ and Yu L: Optimization of extraction and antioxidant activity of polysaccharides from Salvia miltiorrhiza Bunge residue. Int J Biol Macromol 79: 533-541, 2015.

13. Zhou X, Cheung CM, Yang JM, Or PM, Lee WY and Yeung JH: Danshen (Salvia miltiorrhiza) water extract inhibits paracetamolinduced toxicity in primary rat hepatocytes via reducing CYP2E1 activity and oxidative stress. J Pharm Pharmacol 67: 980-989, 2015.

14. Huang M, Wang P, Xu S, Xu W, Xu W, Chu K and Lu J: Biological activities of salvianolic acid B from Salvia miltiorrhiza on type 2 diabetes induced by high-fat diet and streptozotocin. Pharm Biol 53: 1058-1065, 2015.

15. Wang ZS, Luo P, Dai SH, Liu ZB, Zheng XR and Chen T: Salvianolic acid B induces apoptosis in human glioma U87 cells through p38-mediated ROS generation. Cell Mol Neurobiol 33: 921-928, 2013.
16. Yang Y, Ge PJ, Jiang L, Li FL and Zhu QY: Modulation of growth and angiogenic potential of oral squamous carcinoma cells in vitro using salvianolic acid B. BMC Complement Altern Med 11: 54, 2011

17. Wang QL, Wu Q, Tao YY, Liu CH and El-Nezami H: Salvianolic acid $\mathrm{B}$ modulates the expression of drug-metabolizing enzymes in HepG2 cells. Hepatobiliary Pancreat Dis Int 10: 502-508, 2011.

18. Lin C, Liu Z, Lu Y, Yao Y, Zhang Y, Ma Z, Kuai M, Sun X, Sun S, Jing Y, et al: Cardioprotective effect of salvianolic acid B on acute myocardial infarction by promoting autophagy and neovascularization and inhibiting apoptosis. J Pharm Pharmacol 68: 941-952, 2016.

19. Wang JY, Sun J, Huang MY, Wang YS, Hou MF, Sun Y, He H, Krishna N, Chiu SJ, Lin S, et al: STIM1 overexpression promotes colorectal cancer progression, cell motility and COX-2 expression. Oncogene 34: 4358-4367, 2015.

20. Tagliamonte M, Petrizzo A, Tornesello ML, Ciliberto G, Buonaguro FM and Buonaguro L: Combinatorial immunotherapy strategies for hepatocellular carcinoma. Curr Opin Immunol 39: 103-113, 2016

21. Lee JH, Lee Y, Lee M, Heo MK, Song JS, Kim KH, Lee H, Yi NJ, Lee KW, Suh KS, et al: A phase I/IIa study of adjuvant immunotherapy with tumour antigen-pulsed dendritic cells in patients with hepatocellular carcinoma. Br J Cancer 113: 1666-1676, 2015.

22. Babbitt SE, Sutherland MC, San Francisco B, Mendez DL and Kranz RG: Mitochondrial cytochrome $c$ biogenesis: No longer an enigma. Trends Biochem Sci 40: 446-455, 2015.

23. Kulikov AV, Shilov ES, Mufazalov IA, Gogvadze V, Nedospasov SA and Zhivotovsky B: Cytochrome $c$ : The Achilles' heel in apoptosis. Cell Mol Life Sci 69: 1787-1797, 2012.

24. Hao Y, Xie T, Korotcov A, Zhou Y, Pang X, Shan L, Ji H, Sridhar R, Wang P, Califano J, et al: Salvianolic acid B inhibits growth of head and neck squamous cell carcinoma in vitro and in vivo via cyclooxygenase- 2 and apoptotic pathways. Int $\mathbf{J}$ Cancer 124: 2200-2209, 2009.

25. Zhao Y, Guo Y and Gu X: Salvianolic acid B, a potential chemopreventive agent, for head and neck squamous cell cancer. J Oncol 2011: 534548, 2011

26. Ryter SW, Mizumura K and Choi AM: The impact of autophagy on cell death modalities. Int J Cell Biol 2014: 502676, 2014.

27. Levine B and Kroemer G: Autophagy in the pathogenesis of disease. Cell 132: 27-42, 2008.

28. Levine B and Kroemer G: Autophagy in aging, disease and death: The true identity of a cell death impostor. Cell Death Differ 16: $1-2,2009$.

29. Eskelinen EL and Saftig P: Autophagy: A lysosomal degradation pathway with a central role in health and disease. Biochim Biophys Acta 1793: 664-673, 2009.

30. Yang Z and Klionsky DJ: Eaten alive: A history of macroautophagy. Nat Cell Biol 12: 814-822, 2010.

31. Mizushima N, Levine B, Cuervo AM and Klionsky DJ: Autophagy fights disease through cellular self-digestion. Nature 451: 1069-1075, 2008.

32. Kim N, Jeong S, Jing K, Shin S, Kim S, Heo JY, Kweon GR, Park SK, Wu T, Park JI, et al: Docosahexaenoic acid induces cell death in human non-small cell lung cancer cells by repressing mTOR via AMPK activation and PI3K/Akt inhibition. BioMed Res Int 2015: 239764, 2015.

33. Pan WR, Chen YL, Hsu HC and Chen WJ: Antimicrobial peptide GW-H1-induced apoptosis of human gastric cancer AGS cell line is enhanced by suppression of autophagy. Mol Cell Biochem 400: 77-86, 2015

34. Jing Z, Sui X, Yao J, Xie J, Jiang L, Zhou Y, Pan H and Han W: SKF-96365 activates cytoprotective autophagy to delay apoptosis in colorectal cancer cells through inhibition of the calcium/ CaMKII $\gamma /$ AKT-mediated pathway. Cancer Lett 372: 226-238, 2016.

35. Hsin IL, Ou CC, Wu MF, Jan MS, Hsiao YM, Lin CH and Ko JL: GMI, an immunomodulatory protein from Ganoderma microsporum, potentiates cisplatin-induced apoptosis via autophagy in lung cancer cells. Mol Pharm 12: 1534-1543, 2015.

36. Chen L, Meng Y, Sun Q, Zhang Z, Guo X, Sheng X, Tai G, Cheng $\mathrm{H}$ and Zhou Y: Ginsenoside compound $\mathrm{K}$ sensitizes human colon cancer cells to TRAIL-induced apoptosis via autophagy-dependent and -independent DR5 upregulation. Cell Death Dis 7: e2334, 2016.

37. Young MM, Takahashi Y, Khan O, Park S, Hori T, Yun J, Sharma AK, Amin S, Hu CD, Zhang J, et al: Autophagosomal membrane serves as platform for intracellular death-inducing signaling complex (iDISC)-mediated caspase- 8 activation and apoptosis. J Biol Chem 287: 12455-12468, 2012. 
38. Zhang L, Wang K, Lei Y, Li Q, Nice EC and Huang C: Redox signaling: Potential arbitrator of autophagy and apoptosis in therapeutic response. Free Radic Biol Med 89: 452-465, 2015.

39. Lee JJ, Loh K and Yap YS: PI3K/Akt/mTOR inhibitors in breast cancer. Cancer Biol Med 12: 342-354, 2015.

40. Janku F, Kaseb AO, Tsimberidou AM, Wolff RA and Kurzrock R: Identification of novel therapeutic targets in the PI3K/AKT/ mTOR pathway in hepatocellular carcinoma using targeted next generation sequencing. Oncotarget 5: 3012-3022, 2014.

41. Wang F, Mao Y, You Q, Hua D and Cai D: Piperlongumine induces apoptosis and autophagy in human lung cancer cells through inhibition of PI3K/Akt/mTOR pathway. Int J Immunopathol Pharmacol 28: 362-373, 2015.
42. Saito Y, Tanaka Y, Aita Y, Ishii KA, Ikeda T, Isobe K, Kawakami Y, Shimano H, Hara H and Takekoshi K: Sunitinib induces apoptosis in pheochromocytoma tumor cells by inhibiting VEGFR2/Akt/mTOR/S6K1 pathways through modulation of Bcl-2 and BAD. Am J Physiol Endocrinol Metab 302: E615-E625, 2012. 